\title{
Toward Wideband Frequency Rejection: An Efficient Frequency Selective Scheme
}

\author{
V. Nadjari', J. Nourinia', Ch. Ghobadi'1, and M. Majidzadeh² \\ ${ }^{1}$ Department of Electrical Engineering, Urmia University, Urmia, Iran \\ ${ }^{2}$ Department of Electrical and Computer Engineering, Urmia Girls Faculty, West Azarbaijan branch, Technical and Vocational University, Urmia, \\ Iran \\ Corresponding author: V. Nadjari (e-mail: v.nadjari@urmia.ac.ir).
}

\begin{abstract}
This paper outlines a Frequency Selective Surface configuration designed for wideband filtering characteristics. The proposed design topology includes two-layer conductive elements printed on an FR4 substrate with a thickness of $1.6 \mathrm{~mm}$. On the front side, a square loop and four branches are placed, and on the other side of the substrate, there is another square loop supported by two modified vertical arms. The use of square loops as one of the trendy elements in frequency selective designs provides the opportunity to tune the resonances and obtain the desired Performance; The proposed $10 \mathrm{~mm} \times 10 \mathrm{~mm}$ frequency selective surface unit cell structure rejects the frequency band of 2-14 GHz covering WiMAX, WLAN, and X band inservice frequency ranges. It has three resonant frequencies and works well in various incident angles. There is a good match between the simulated results and the tested results. Stable frequency response, simple structure, and easy production are useful features of the developed scheme.
\end{abstract}

INDEX TERMS Frequency selective surface, Square loop, Stable response, Wide rejection region.

\section{INTRODUCTION}

Surface with a periodic alignment of its elements, A which is designed to transmit, reflect, or absorb distinctive frequency ranges, is called Frequency Selective Surface (FSS). A single element, namely a unit cell, repeats in many rows and columns to form the final periodic structure. Each FSS, depending on its unit cell type, could be designed to pass or reject desired frequency bands. The distance of the unit cell from each other, their size, and arrangement are essential factors to adjust the exciting resonance and operation bands. The common elements used in FSS designs are arranged into four groups:

1) The center connected or N-poles

2) The loop types

3) Solid interior or plate type of various shapes

4) Combinations

Fig. 1 shows all four groups [1].

Owing to their impressive and marvelous characteristics, this category of filters is now widely used in every aspect of the communication industry. They have found applications in many diverse branches. For instance, in [2], an ultrathin FSS design with polarization-independent performance is designed for stealth technology. The suggested structure is composed of a square unit cell loaded with three-ring slot pairs. The obtained passband for the FSS is from $33 \mathrm{GHz}$ to $37.5 \mathrm{GHz}$ and flat. The use of this structure is in stealth technology applications in the Ka frequency band. As another example of such stealth technology applications is an FSS-based hybrid nanocomposite absorber with enhanced bandwidth for stealth applications proposed in [3]; Moreover, we could find some examples of absorbing applications of FSS structures in [4-5]. Other applications include antenna radome [6-5], radar cross section (RCS) reduction [8-9], multi-band antennas and polarization rotator [10-11] electromagnetic shielding [12-13], and beamforming applications [14-15]. All these researches have released valuable and exciting results, but the day by day update of the communication industry calls the need for more efficient and reliable structures.

Targeting greater improvements in FSS design, this paper proposes a novel structure of FSS unit cell for wideband rejection applications. The square loop is the primary element in this alignment, picked for both the front side and backside of the substrate. On the front side, four branches connect to the square loop. On the backside, we embedded two vertical arms with a modified structure at their joint points to the square loop. The suitably tuned and cited conductive elements result in wideband rejection from $2 \mathrm{GHz}$ to $14 \mathrm{GHz}$. The bandwidth of any FSS can be varied significantly by variation of the inter-element spacing, a larger spacing will, in general, produce a narrower bandwidth, and vice versa. The dominant advantages of developed sketch could be listed as follows:

- Proposing a highly reliable FSS scheme suitable for wideband rejection.

- Using simple FSS elements on the front side and backside.

- Stable frequency response and close performance validation through both simulated and measured processes. 
This FSS is designed and analyzed through Ansys HFSS software, which uses the finite-element method. It has goldstandard accuracy, advanced solver and high-performance compute technology. We organized this manuscript as follows: section II goes into Unit Cell topology and performance. Section III compares the proposed structure with other similar structures and discusses its advantages. Section IV examines the surface current distribution. FSS performance against different angles of incidence was carried out in section V to show the stability of it. Section VI shows the FSS measurement process and comparison of the simulated and experimental results. Finally, section VII concludes the paper.

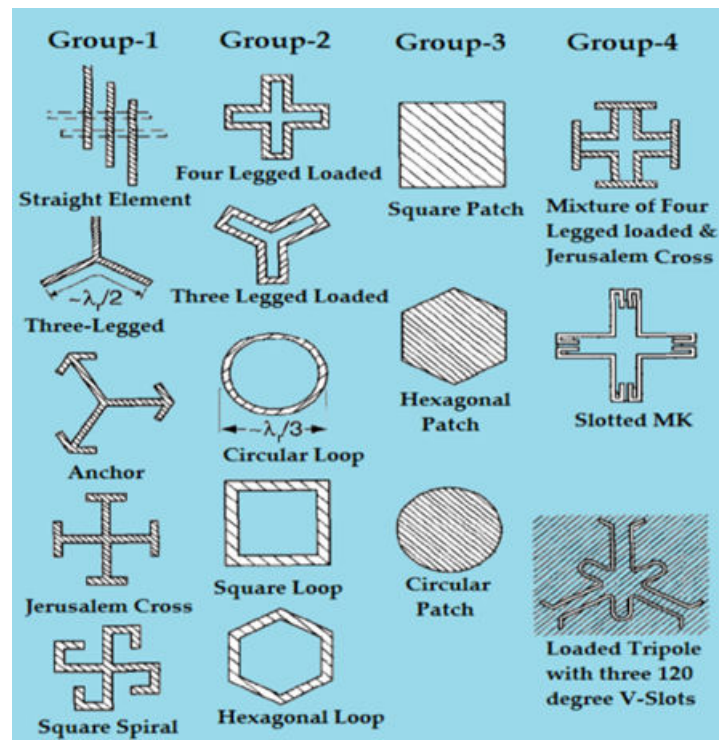

FIGURE 1. typical element types arranged in groups [1].

\section{UNIT CELL CONFIGURATION AND PERFORMANCE}

Fig. 2 depicts the constituent element as the unit cell of the presented FSS in detail. As we can see in the top view of the cell, there is a square loop with four arms attached. On the other side, we put another square loop with two arms connected to it. For increasing the functionalities of the FSS, the jointing points of the arms and square loops are modified. The single unit cell is printed on a $10 \mathrm{~mm} \times 10 \mathrm{~mm}$ FR4 substrate whose thickness is $1.6 \mathrm{~mm}$. The square loop is the primary building block of the proposed design. As a wellknown fact, this element is one of the most applicable and easy to use elements in FSS configurations, which will be discussed later in this paper.

Evolving an efficient unit cell requires many factors altogether that we should consider. Herein, the design process of the unit cell is explored in five steps, as seen in Fig. 3. In each step, suitable modifications have yield improvements in FSS performance. As can be seen, in step 1, only one square loop is embedded on the front side, and there is no conductive element on the backside. According to the presented results in Fig. 4, this formation rejects $2.5-5 \mathrm{GHz}$ with the central frequency at $3.8 \mathrm{GHz}$. In step 2, a similar square loop with some different dimensions is adopted on the backside too. As each loop excites a resonance, we estimate that two resonances should be present in frequency response. The results in Fig. 4 confirm this estimation. As the dimensions of the loops are selected close to each other, the exciting resonances are very close that this configuration rejects 1.8-6 GHz. In step 3, there are four branches on the front side of the substrate, attached to the square loop. This modification excites another resonance at $13.3 \mathrm{GHz}$, which yields a two-band operation. The modifications in step 4 have shifted the upper band resonance to $11.8 \mathrm{GHz}$. It is worth noting the lower frequency range is almost constant whereas the upper band rejected band has been widened. Finally, in step 5, which is the final design, the front side and the backside of the layer are finalized. We put two vertical arms on the backside. The last two steps in the design process help us control several bands, especially the Xband, in the future design of this structure called the tunable FSS. We explain more about it later.

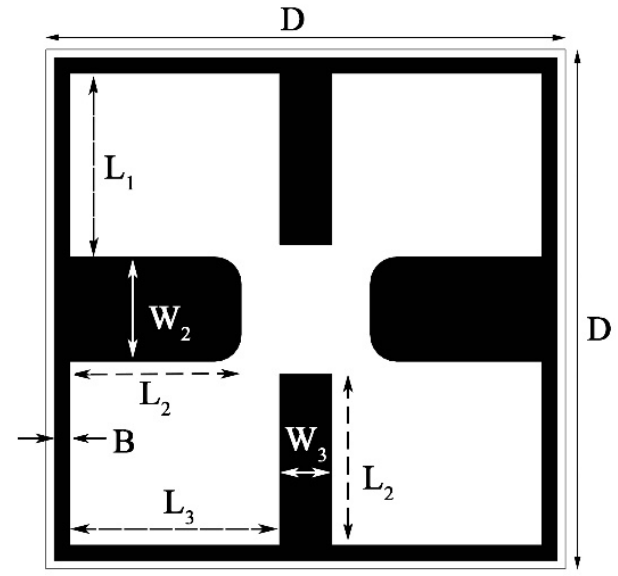

(a)

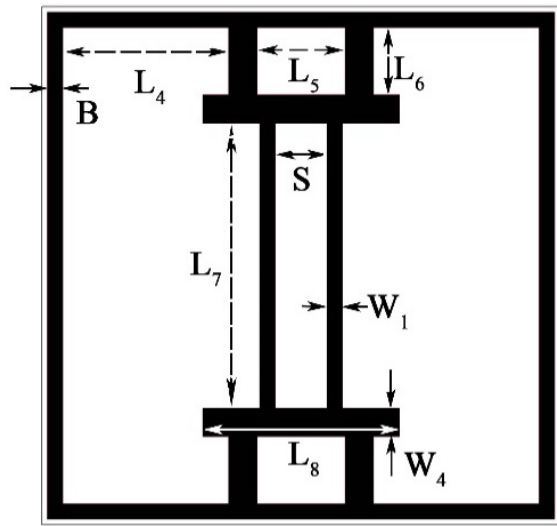

(b)

FIGURE 2. Schematic view of the proposed FSS unit cell. (a) front side, (b) backside.

The proposed final design blocks the wide frequency band of 2-14 GHz. Proper positioning of elements and optimizing their dimensions make the designed structure an acceptable 
one for wideband filtering applications in communication systems. Here, we intend to examine the effect of the two parameters, (S) and (B), on the structure performance. According to the presented results in Fig. 5(a), as the (S) parameter increases, a new band appears from $2.2 \mathrm{GHz}$ to 3.4 $\mathrm{GHz}$, and the second and third resonances shift to higher frequencies due to the increase in capacitance. However, decreasing the $(\mathrm{S})$ parameter eliminates the lower resonance and creates two bands from 2.2-8.9 GHz and 10.3-13 GHz. As shown in fig. 5(b), increasing the (B) parameter shifts all the resonance frequencies to higher frequencies, and Decreasing it shifts the resonances to lower frequencies slightly and yields two frequency bands from 2.2-3.2 GHz and 3.8-13.9 GHz. We manufactured a prototype of the proposed structure in the dimensions of $200 \mathrm{~mm} \times 200 \mathrm{~mm}$ as we see in Fig. 6 .

TABLE I. Dimensions of the FSS unit cell

\begin{tabular}{cc||cc}
\hline \hline Parameter & Dimension(mm) & Parameter & Dimension(mm) \\
\hline $\mathrm{D}$ & 10 & $\mathrm{~L}_{2}$ & 3.3 \\
$\mathrm{~B}$ & 0.3 & $\mathrm{~L}_{3}$ & 4.05 \\
$\mathrm{~W}_{1}$ & 0.3 & $\mathrm{~L}_{4}$ & 3.2 \\
$\mathrm{~W}_{2}$ & 2 & $\mathrm{~L}_{5}$ & 1.7 \\
$\mathrm{~W}_{3}$ & 1 & $\mathrm{~L}_{6}$ & 1.3 \\
$\mathrm{~W}_{4}$ & 0.55 & $\mathrm{~L}_{7}$ & 5.5 \\
$\mathrm{~S}$ & 1 & $\mathrm{~L}_{8}$ & 3.8 \\
$\mathrm{~L}_{1}$ & 3.55 & & \\
\hline \hline
\end{tabular}

(a)

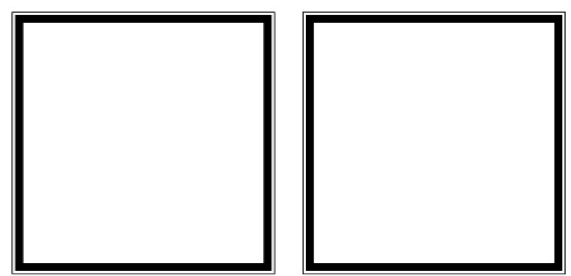

(b)
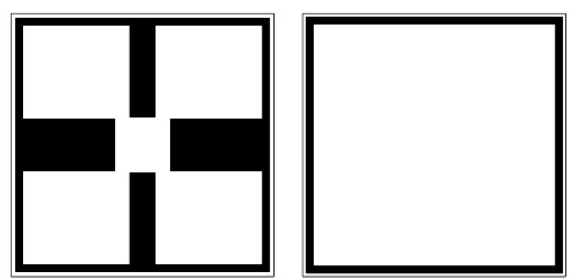

(c)

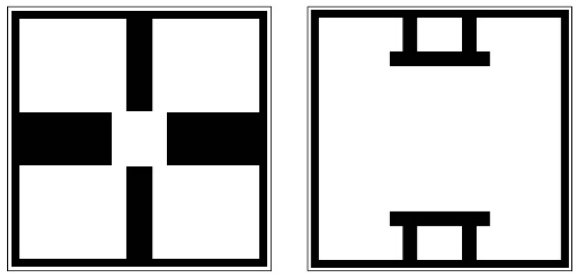

(d)
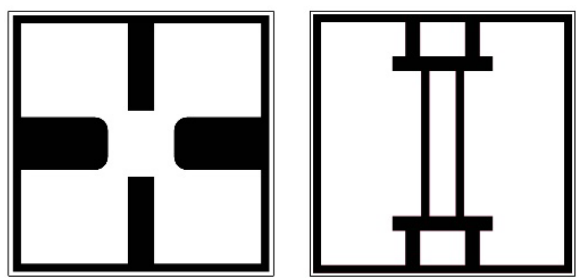

(e)

FIGURE 3. Five steps in FSS design. (a) step 1, (b) step 2, (c) step 3, (d) step 4, (e) step 5.

Further discussion on the proposed FSS could be reached through the equivalent circuit analysis. This analysis method is an efficient way to mathematically confirm how different resonances are excited by different elements adopted in the FSS design. For the first steps of design, the governing mathematical formulations regarding the square loop are followed [20]:

$$
\begin{aligned}
& B_{C}=\epsilon_{\text {eff }} \frac{4}{Z_{0}} \frac{d}{p} F(p, g, \lambda) \\
& X_{L}=Z_{0} \frac{d}{p} F(p, 2 B, \lambda)
\end{aligned}
$$

Where $\mathrm{F}$ is as follows:

$$
F(p, w, \lambda)=\frac{p}{\lambda} \cos \theta\left[\operatorname{Ln}\left(\operatorname{cosec} \frac{\pi B}{2 p}\right)+G(p, B, \lambda)\right]
$$

And $\mathrm{G}$ is:

$G(p, B, \lambda)=\frac{1}{2} \frac{\left(1-\beta^{2}\right)^{2}\left[\left(1-\frac{\beta^{2}}{4}\right)\left(A_{+}+A_{-}\right)+4 \beta^{2} A_{+} A_{-}\right]}{\left(1-\frac{\beta^{2}}{4}\right)+\beta^{2}\left(1+\frac{\beta^{2}}{2}-\frac{\beta^{4}}{8}\right)\left(A_{+}+A_{-}\right)+2 \beta^{6} A_{+} A_{-}}$

In which:

$$
\begin{aligned}
& A_{ \pm}=\frac{1}{\sqrt{1 \pm \frac{2 p \sin \theta}{\lambda}-\left(\frac{p \cos \theta}{\lambda}\right)^{2}}}-1 \\
& \beta=\frac{\sin \pi B}{2 p} \\
& \epsilon_{e f f}=\epsilon_{r}+\left(\epsilon_{r}-1\right) \cdot\left[\frac{-1}{e^{x \cdot N}}\right]
\end{aligned}
$$

Where

$$
x=10 \frac{t}{p}
$$

It should be mentioned that in the above formulas, $B_{c}$ is the capacitive susceptance, $X_{L}$ is the inductive reactance, $\mathrm{p}$ is the periodicity, $g$ is the gap between the loop sides, $\lambda$ is the wavelength, $\theta$ is the angle of incidence, $t$ is the thickness of 
the substrate, and $\mathrm{N}$ is an exponential factor that varies with the geometry of the unit cell. For square-loop arrays, $\mathrm{N}$ is around 1.8, and for metal patches $\mathrm{N}=1.3$. We use the following equations for the arms [21]:

$$
\begin{aligned}
& L_{A}=\frac{\mu_{0}}{2 \pi} \frac{1}{w^{2}}\left[\operatorname{lw^{2}} \operatorname{Ln}\left(\frac{1}{w}+\sqrt{\left(\frac{l}{w}\right)^{2}+1}\right)+\right. \\
& l^{2} w \operatorname{Ln}\left(\frac{w}{l}+\sqrt{\left(\frac{w}{l}\right)^{2}+1}\right)+\frac{1}{3}\left(l^{3}+w^{3}\right)- \\
& \left.\frac{1}{3}\left(l^{2}+w^{2}\right)^{\frac{3}{2}}\right] \\
& C_{A}=\frac{\sqrt{\epsilon_{e f f}}}{2 c z_{0}}-\epsilon_{0} \epsilon_{r} \frac{w}{2 h}
\end{aligned}
$$

Which $\mathrm{c}$ is the speed of light, and $\mathrm{Z}_{0}$ is the characteristic impedance of the arms. In the final step, the inductance and capacitance of the parallel strips can be obtained by [22]:

$$
\begin{aligned}
& L_{p}=\frac{\mu_{0}}{2 \pi}\left(l \sin h^{-1} \frac{l}{s}-\sqrt{l^{2}+S^{2}}+S\right) \\
& C_{p}=\frac{\sqrt{\epsilon_{e f f}}}{2 c z_{0}}+\epsilon_{0} \epsilon_{r} \frac{w}{2 h}+C_{f}^{\prime} \\
& C_{f}^{\prime}=\frac{C_{A}}{1+\left(A \frac{h}{3}\right) \tan h\left(\frac{8 S}{h}\right)} \sqrt{\frac{\epsilon_{r}}{\epsilon_{e} f f}} \\
& A=\exp \{-0.1 \exp (2.33-2.53 \mathrm{w} / h)\}
\end{aligned}
$$

Therefore, the resonance frequency can be obtained by the following equation:

$$
f_{r}=\frac{1}{2 \pi \sqrt{L C}}
$$

\section{COMPARISON}

In this section, we have compiled a table to compare the proposed FSS with similar structures. We selected the FSS structures in [13] and [16]- [19] for this purpose. The factors that we have chosen to compare include the unit cell size, bandwidth, number of substrates, ability to filter $802.16 \mathrm{~d}$ WiMAX, and 802.16e WiMAX, 802.11 WLAN (except 900 $\mathrm{MHz}$ and $60 \mathrm{GHz}$ ), and X-band. In terms of size, this structure and [16], is the smallest compared to others, but [16] does not support WLAN and WiMAX bands except for $5.8 \mathrm{GHz}$ for WiMAX and 4.9, 5, and $5.9 \mathrm{GHz}$ for WLAN. Among these, [19] is better than the others and covers the desired bands except for 2.4GHZ WLAN and 2.3 and $2.5 \mathrm{GHz}$ WiMAX. However, we should not forget that [19] has two substrate layers. [17] and [18] are more extensive and do not meet the bandwidth requirements. Here it is clear that the proposed structure supports all the usual bands we have in mind despite being single-substrate and small in size. Although there is no comprehensive research on the effects of these bands on human health, however, the proposed structure is useful in filtering them.

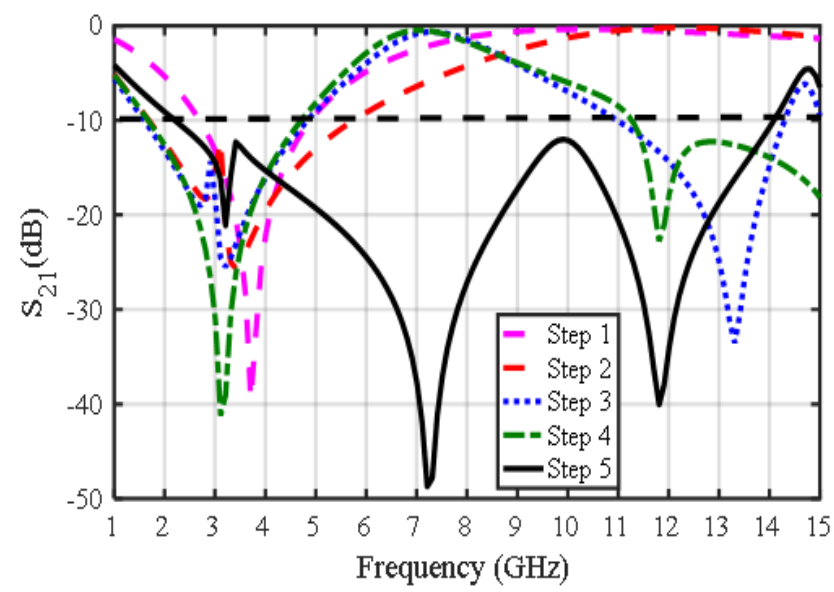

FIGURE 4. $\mathrm{S}_{21}$ curves for the FSS unit cells in the design process.

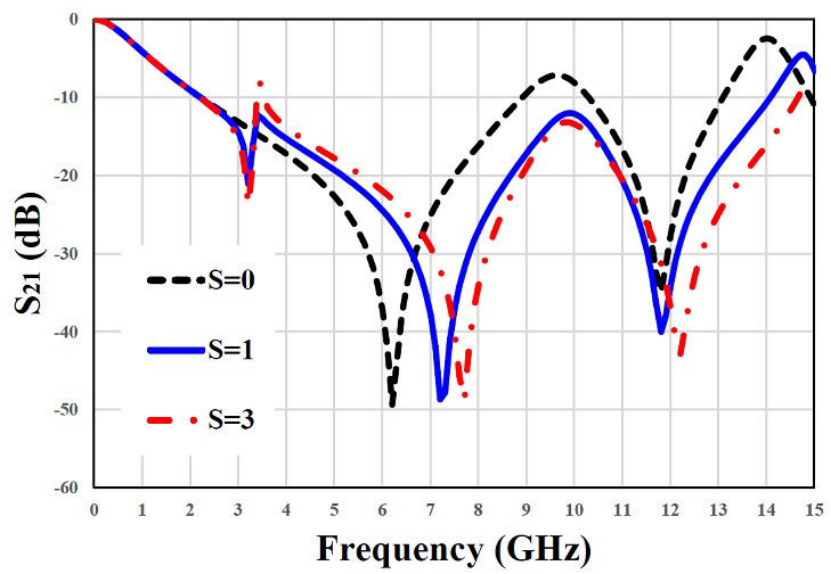

(a)

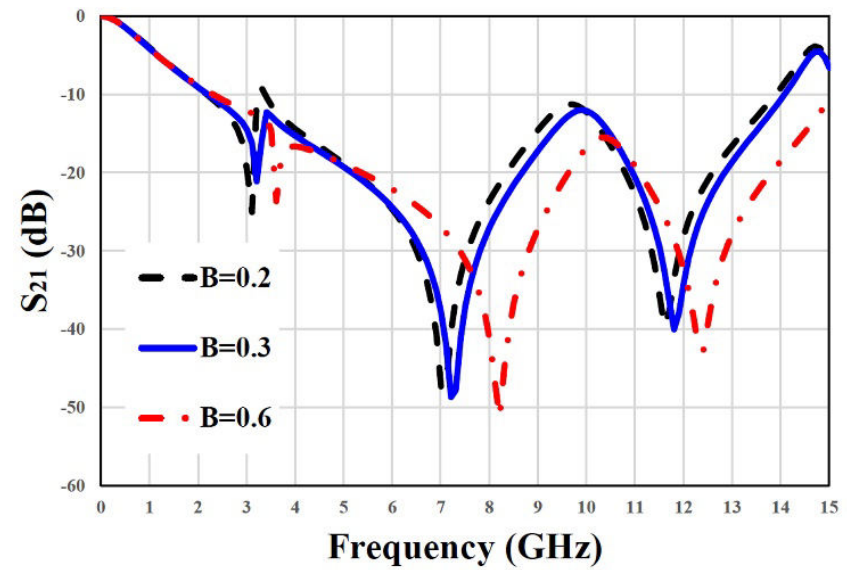

(b)

FIGURE 5. $S_{21}$ curves for (a) $S$ parameter, and (b) B parameter 
TABLE II. Comparison of the proposed structure with other wideband structures.

\begin{tabular}{|c|c|c|c|c|c|c|c|c|c|c|}
\hline $\begin{array}{l}\text { D } \\
\text { D } \\
\frac{\mathbb{D}}{\mathbb{D}} \\
\frac{\mathbb{D}}{D} \\
\stackrel{D}{D}\end{array}$ & $\begin{array}{l}\frac{n}{N} \\
\frac{D}{3} \\
3 \\
3 \\
w\end{array}$ & 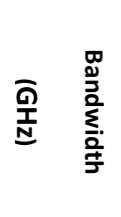 & 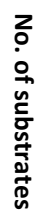 & $\frac{\sum}{3}$ & $\begin{array}{l}\infty \\
\stackrel{\infty}{0} \\
\stackrel{\circ}{2}\end{array}$ & 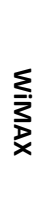 & $\begin{array}{l}\infty \\
\stackrel{\infty}{0} \\
\stackrel{\sigma}{D}\end{array}$ & $\frac{5}{2}$ & $\stackrel{\infty}{\stackrel{\infty}{N}}$ & 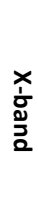 \\
\hline 13 & $\begin{array}{l}12 \times 12 \\
\times 3.2\end{array}$ & $\begin{array}{c}6.5- \\
14\end{array}$ & 1 & & $x$ & & $x$ & & $x$ & $\checkmark$ \\
\hline 16 & $\begin{array}{l}10 \times 10 \\
\times 1.6\end{array}$ & $\begin{array}{c}4.6- \\
16\end{array}$ & 1 & & $x$ & & $x$ & & $x$ & $\checkmark$ \\
\hline 17 & $\begin{array}{l}22 \times 22 \\
\times 1.6\end{array}$ & $\begin{array}{l}3.7- \\
6.23\end{array}$ & 1 & & $x$ & & $x$ & & $x$ & $x$ \\
\hline 18 & $\begin{array}{l}20 \times 20 \\
\times 0.48\end{array}$ & $\begin{array}{l}8.99- \\
11.03\end{array}$ & 1 & & $x$ & & $x$ & & $x$ & $x$ \\
\hline 19 & $\begin{array}{l}15 \times 15 \\
\times 1.5\end{array}$ & $\begin{array}{l}2.7- \\
13.2\end{array}$ & 2 & & $\checkmark$ & & $x$ & & $x$ & $\checkmark$ \\
\hline $\begin{array}{l}\text { Prop } \\
\text { osed } \\
\text { FSS }\end{array}$ & $\begin{array}{l}10 \times 10 \\
\times 1.6\end{array}$ & $2-14$ & 1 & & $\checkmark$ & & $\checkmark$ & & $\checkmark$ & $\checkmark$ \\
\hline
\end{tabular}

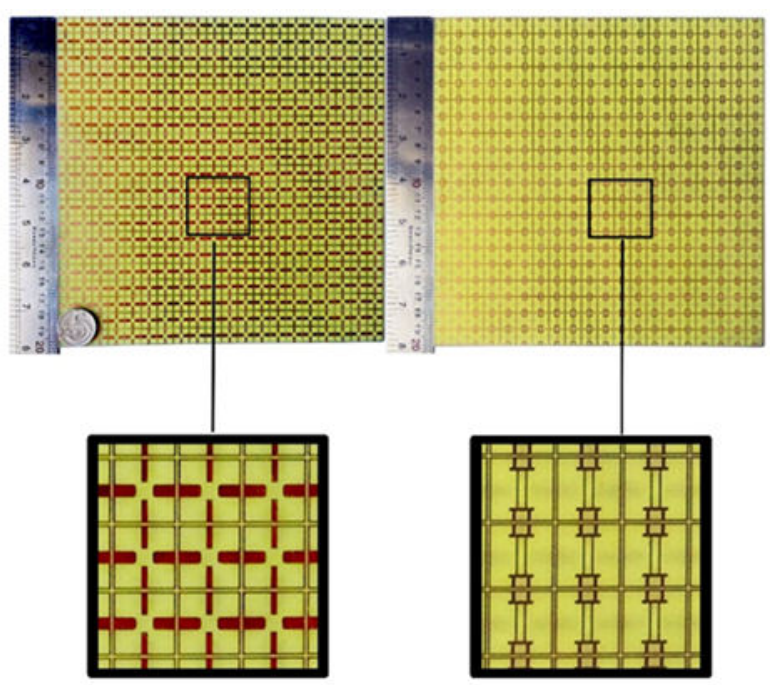

FIGURE 6. Fabricated prototype photograph

\section{SURFACE CURRENT DISTRIBUTION ANALYSIS}

For further studying, we analyzed the distribution of surface current at resonance frequencies on the FSS unit cell. Fig. 7 depicts the currents on the front and backside of the FSS unit cell. As mentioned before, three main resonances are excited in the $\mathrm{S}_{21}$ curve. At $3.2 \mathrm{GHz}$, which is the first excited resonance, the front side square carries the primary radiating current. We concluded that this element is the most responsible one for the emergence of the resonance. Then, for the second resonance at $7.2 \mathrm{GHz}$, the current is focused on the backside arms extended from the top to bottom of the square loop. Also, at $11.8 \mathrm{GHz}$, the last resonance, the arms on the front side of the substrate play the most crucial role. The suitable arrangement of the conductive elements and exciting each resonance by one of the adopted elements results in a broadband frequency rejection.

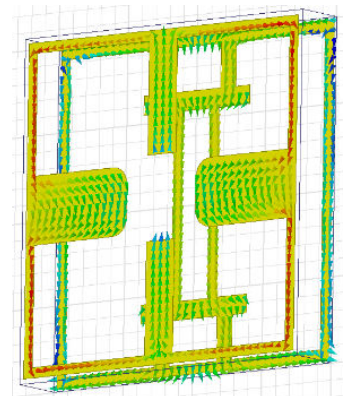

(a)

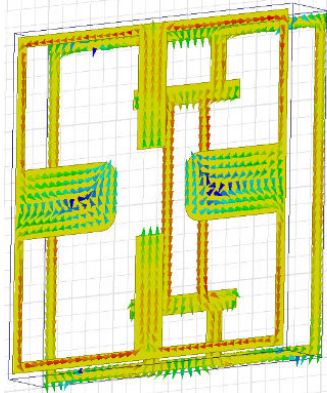

(b)

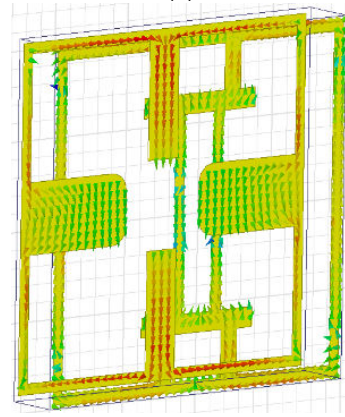

(c)

FIGURE 7. Distribution of the surface current at (a) $3.2 \mathrm{GHz}$, (b) $7.2 \mathrm{GHz}$, (c) $11.8 \mathrm{GHz}$.

\section{SENSITIVITY ANALYSIS}

One main point to be considered in FSS design is its ability to performing in different working conditions. It means illuminating signals with various incident angles on it, whereas having almost the same function. This section discusses this aspect of FSS. Fig. 8 depicts the FSS unit cell performance for incident angles up to 50 degrees. We have only set the angle from 0 to 50 degrees to avoid chart junk, but it has been tested in experiments up to 70 degrees. As shown in Fig. 8, highly reliable performance is observed for the proposed design. Also, filleting the corners of more massive 
arms in step 5 of the design effectively stabilizes the response at an angle of 10-20 degrees.

\section{RESULT AND DISCUSSION}

Surely a designed FSS unit cell needs to be tested in a real experimental position to evaluate its reliability in real applications. To this end, the proposed FSS is fabricated and tested in an antenna and microwave laboratory. Fig. 9 demonstrates the FSS test setup. The measurement setup for the designed FSS includes two ultra-broadband horn antennas (frequency range of 1-18GHz), each on the opposite sides of the structure, which has high gain and low VSWR across its operational frequency band. These antennas maintain a single main lobe pattern in the direction of the horn axis over its frequency range. We benefit from uniform illumination of target surfaces and accurate measurement. The network analyzer is an Agilent technology E8363C PNA. Like the simulation process, we used one antenna as a transmitter, which illuminates a wide range of frequencies toward the FSS. The FSS passband allows the signals which are in the passband region to pass and gathered by the receiver antenna whereas those which are not in the specific region are rejected. The fabricated prototype is surrounded by RF Absorbing Materials (RAM) to avoid spillover or diffraction at the edges of the structures, although a bigger size of the FSS prototype is recommended. To simulate a quiet environment, we tested the fabricated prototype in the anechoic chamber. Fig. 10 compares the measured performance with the simulated one. We obtained a cooperative agreement between the results, which ensure the FSS proper performance. The proposed structure can be used as a shielding structure, although our goal of achieving such bandwidth is to use it as a tunable structure for the next attempts. By placing PIN diodes on the pre-specified locations on the structure, a tunable structure can be achieved, which, depending on whether the diodes are in ON or OFF state, we can achieve the demanding bands.

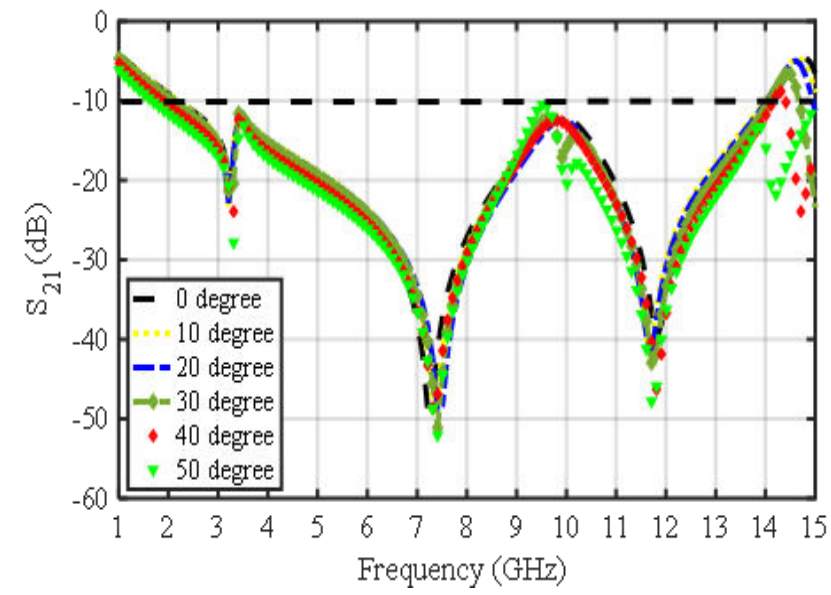

FIGURE 8. FSS performance for different incident angles.

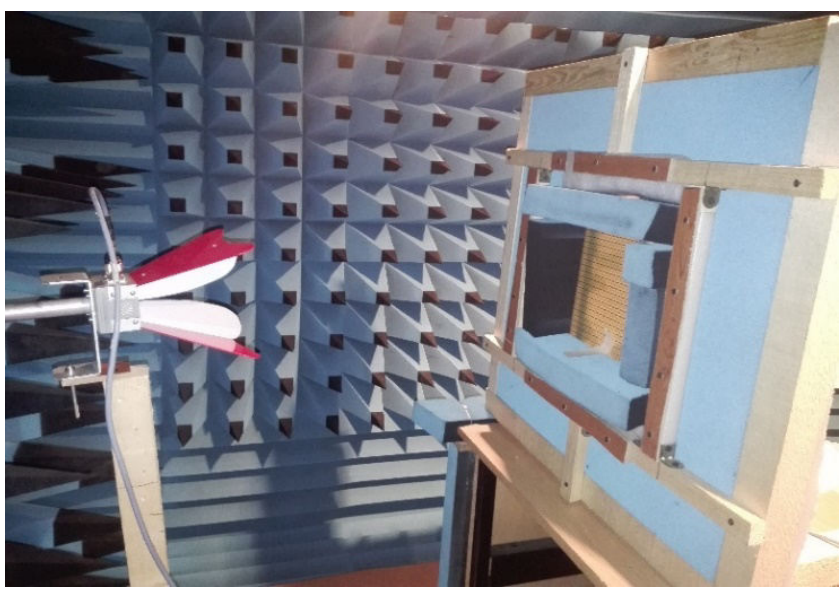

FIGURE 9. FSS in the measurement process

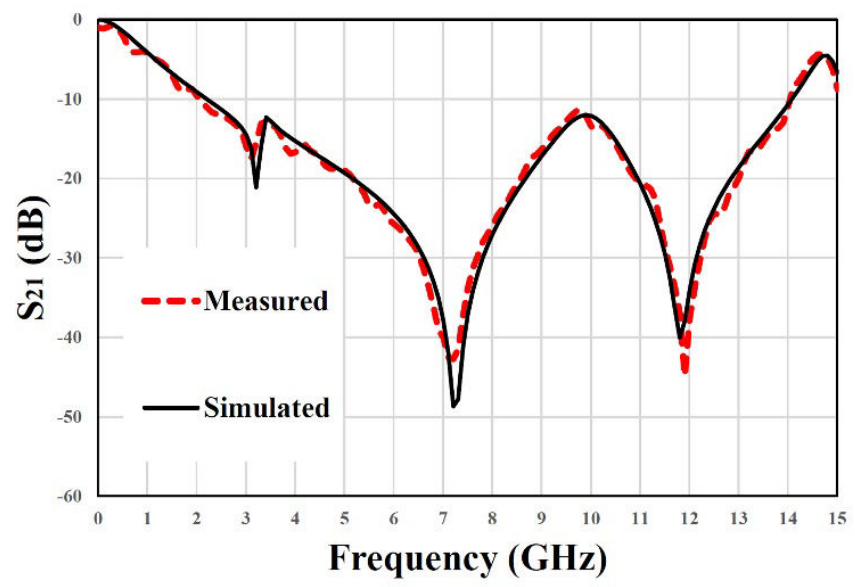

FIGURE 10. Comparison of simulated and measured $S_{21}$ curves for the proposed FSS

\section{CONCLUSION}

A novel scheme of an FSS unit cell was designed, analyzed, and measured. Ansys HFSS was used in the simulation and analysis processes. It was shown that the adoption of the square loop, along with some other modifications, yields a wideband rejection from 2-14 GHz. A parametric study has been done in which one parameter was changed at a time, whereas others were kept fixed. The stability of the response against various incident angles was analyzed. It was recognized that the proposed FSS exhibit almost constant response up to a 50 degrees' incident angle. Moreover, simple conductive elements make the structure cost-effective and simple configure, which could be easily used in filtering applications. The efficiency of the proposed FSS was validated in the real experimental position in the microwave lab. The forthcoming works will be on turning this structure into a tunable FSS employing PIN diodes. The use of this upcoming tunable FSS will be in the filtenna systems. 


\section{REFERENCES}

[1] B. A. Munk, Frequency selective surface: Theory and design. 1st ed., New York, NY: Wiley-Interscience, 2000, pp. 1-78.

[2] W. Wu, X. Liu, K. Cui, et al., "An ultrathin and polarization-insensitive frequency selective surface at Ka-band," IEEE Antennas Wireless Propag. Lett., vol. 17, no. 1, pp. 74-77, 2018, 10.1109/LAWP.2017.2774825.

[3] V. K. Chakradhary, H. B. Baskey, R. Roshan et al, "Design of frequency selective surface-based hybrid nanocomposite absorber for stealth applications," IEEE Transactions on Microwave Theory and Techniques, vol. 66, no. 11, pp. 4737-4744, 2018 10.1109/TMTT.2018.2864298.

[4] Q. Zhou, P. Liu, D. Yu, et al., "Field-controlled switchable frequency selective surface with broadband absorption characteristic,". IET Microwaves, Antennas \& Propagation, vol. 12, no. 9, pp. 1470-1476, 2018, 10.1049/iet-map.2017.0818.

[5] Q. Yuan, H. Ma, A. Sui, et al., "A broadband wide-angle synthetical absorber designed by topology optimization of resistance surface and metal wires," IEEE Access., vol. 7, pp. 142675-142681, 2019, 10.1109/ACCESS.2019.2942495.

[6] B. Gao, S. Huang, Z. Ren, et al., "Design and verification of an integrated free-standing thick-screen FSS radome," IEEE Antennas Wireless Propag. Lett., vol. 17, no. 9, pp. 1630-1634,2018, 10.1109/LAWP.2018.2859232.

[7] N. Liu, X. Sheng, C. Zhang, et al., "Design of frequency selective surface structure with high angular stability for radome application," IEEE Antennas Wireless Propag Lett., vol. 17, no. 1, pp. 138-141, 2018, 10.1109/LAWP.2017.2778078.

[8] H. Huang, Z. Shen, "Low-RCS reflectarray with phase controllable absorptive frequency-selective reflector," IEEE Transactions on Antennas and Propag., vol. 67, no. 1, pp. 190-198, 2019, 10.1109/TAP.2018.2876708.

[9] M. Pazokian, N. Komjani, M. Karimpour, "Broadband RCS reduction of microstrip antenna using coding frequency selective surface," IEEE Antennas Wireless Propag. Lett., vol. 17, no. 8, pp. 1382-1385, 2018, 10.1109/LAWP.2018.2846613.

[10] S. Sah, M. R. Tripathy, A. Mittal, "Multiband and Miniaturized dual layer Antenna incorporated with FSS and DGS," Advanced Electromagnetics, vol. 7, no. 1, pp. 1-6, 29 Jan. 2018, 10.7716/aem. v7i1.534

[11] K. K. Varikuntla, R. Singarav, "Review on Design of Frequency Selective Surfaces based on Substrate Integrated Waveguide Technology," Advanced Electromagnetics, vol. 7, no. 5, pp. 101-110, Nov. 2018, 10.7716/aem. v7i5.751

[12] W. Yin, H. Zhang, T. ZHONG, et al., "A novel compact dual-band frequency selective surface for GSM shielding by utilizing a 2.5-dimensional structure," IEEE Transactions on Electromagnetic Compatibility, vol. 60, no. 6, pp. 2057-2060, 2018, 10.1109/TEMC.2018.2790584.

[13] I. S. Syed, Y. Ranga, L. Matekovits, et al., "A single-layer frequency-selective surface for ultrawideband electromagnetic shielding," IEEE Transactions on Electromagnetic Compatibility, vol. 56, no. 6, pp. 14041411, 2014, 10.1109/TEMC.2014.2316288.
[14] G. H. Elzwawi, H. H. Elzwawi, M. M. Tahseen, et al., "Frequency selective surface-based switchedbeamforming antenna," IEEE Access., vol. 6, pp. 4804248050, 2018, 10.1109/ACCESS.2018.2850808.

[15] Y. Youn, S. Cho, H. Song, et al., "Polarization and frequency-selective surface for vehicular beamforming communications requiring near-zero profile," IEEE Trans. on Vehicular Technol., 2020, vol. 69, no. 2, pp. 17191726, 2020, 10.1109/TVT.2019.2959376.

[16] M. Majidzadeh, C. Ghobadi, and J. Nourinia, "Ultrawide band electro-magnetic shielding through a simple single layer frequency selective surfaces," Wireless Pers. Commun., vol. 95, pp. 2769-2783, 2017.

[17] G. Sen, S. Mahato, T. Mandal, S. Mondal, S. Majumdar, and P. P. Sarkar, "Design of a wideband frequency selective surface (FSS) for multiband operation of reflector antenna," In 5th International conference on computers and devices for communication (CODEC), 2012, pp. 1-3.

[18] F. Guan, H. Xiao, M. Shi, and F. Wang, "The novel frequency selective fabric and application research," Journal of Industrial Textile, vol. 46, 2015, pp. 143-159.

[19] H. F. Huang, Sh. F. Zhang, and Y. H. Hu, "A novel frequency selective surface for ultra-wideband antenna performance improvement," In Proceedings of the international symposium on antennas \& propagation (ISAP), 2013, pp. 965-968.

[20] R. J. Langley, E. A. Parker, "Equivalent circuit model for arrays of square loops," Electronics Letters, vol. 18, 1982, pp. 294-296.

[21] R. P. Clayton, Inductance: loop and partial. $1^{\text {st }}$ ed., NJ, Wily \& Sons, Inc., 2010, Ch. 6, pp. 245-264

[22] T. C. Edwards, Foundation for microstrip circuit design. $2^{\text {nd }}$ ed., 1991 , pp. $237-298$ 